VOL. $76(2007)$ [155-160]

\title{
A NOTE ON BOUNDED VARIATION AND HEAT SEMIGROUP ON RIEMANNIAN MANIFOLDS
}

\author{
A. Carbonaro and G. Mauceri
}

\begin{abstract}
In a recent paper Miranda Jr., Pallara, Paronetto and Preunkert have shown that the classical De Giorgi's heat kernel characterisation of functions of bounded variation on Euclidean space extends to Riemannian manifolds with Ricci curvature bounded from below and which satisfy a uniform lower bound estimate on the volume of geodesic balls of fixed radius. We give a shorter proof of the same result assuming only the lower bound on the Ricci curvature.
\end{abstract}

\section{INTRODUCTION}

Functions of bounded variation in $\mathbb{R}^{n}$ have an important role in several classical problems of calculus of variations. Indeed a very important particular case of $B V$ functions are characteristic functions of sets with finite perimeter in the sense of Caccioppoli-De Giorgi, the most natural class of sets where the isoperimetric problem can be formulated and solved. More recently this class of functions has been the natural tool to study problems where discontinuity hypersurfaces appear as, for instance, in image segmentation theory and fracture mechanics $[\mathbf{1}]$.

After earlier attempts by Tonelli and Cesari to generalise to several variables the classical notion of bounded variation for functions of one variable, the modern theory of $B V$ functions was tied to distributions by the work of Fichera [5] and De Giorgi $[3,4]$. In particular De Giorgi's definition is based on a property of the heat semigroup approximations $\left\{P_{t} u: t>0\right\}$ of a function $u$. Namely in $[3,4]$ he proved that if $u \in L^{\infty}\left(\mathbb{R}^{n}\right)$ then the limit

$$
I(u)=\lim _{t \rightarrow 0}\left\|\nabla P_{t} u\right\|_{L^{1}\left(\mathbf{R}^{n}\right)}
$$

exists and is finite if and only the distributional gradient of $u$ is an $\mathbb{R}^{n}$-valued measure $D u$ with finite total variation $|D u|$. Moreover, if this is the case, then $I(u)=|D u|\left(\mathbb{R}^{n}\right)$. Notice that, if $u$ is in $L^{1}\left(\mathbb{R}^{n}\right)$ then

$$
|D u|\left(\mathbb{R}^{n}\right)=\sup \left\{\int_{\mathbf{R}^{n}} u \operatorname{div} g \mathrm{~d} x: g \in C_{c}^{1}\left(\mathbb{R}^{n} ; \mathbb{R}^{n}\right),\|g\|_{\infty} \leqslant 1\right\} .
$$

\section{Received 24th January, 2007}

Work partially supported by the MIUR PRIN 2005 "Analisi Armonica".

Copyright Clearance Centre, Inc. Serial-fee code: 0004-9727/07 \$A2.00+0.00. 
This last formula, first introduced by Miranda in [6], is now used to define $B V$ functions, and can be easily generalised to a Riemannian manifold $M$. It is natural to ask if the equality

$$
|D u|(M)=\lim _{t \rightarrow 0}\left\|\mathrm{~d} P_{t} u\right\|_{1}
$$

holds in this context, where the left hand side is defined by a Riemannian counterpart of (1.2) (see (3.1) below) and in the right hand side $d$ denotes exterior differentiation and $\left(P_{t}\right)_{t \geqslant 0}$ is the heat semigroup generated by the Laplace-Beltrami operator on $M$.

In a recent paper [7] Miranda Jr., Pallara, Paronetto and Preunkert proved that (1.3) does indeed hold in a complete, connected Riemannian manifold satisfying the following two geometric assumptions

(H1) the Ricci curvature is bounded from below;

(H2) the volume of geodesic balls of fixed radius has a positive lower bound which does not depend on the centre.

Both assumptions are heavily used to obtain Gaussian estimates of the heat kernel and its covariant derivative.

In this note we present a shorter proof of this result, which does not need assumption (H2). Our proof avoids the use of Gaussian estimates of the heat kernel and is based on some simple properties of the heat semigroup generated by the de Rham Laplacian on differential forms.[2].

An example of a Riemannian manifold with Ricci curvature bounded from below which does not satisfy assumption (H2) is a warped product of $d$-dimensional Euclidean space $\mathbb{R}^{d}$ with the 1-dimensional torus $\mathbb{T}$, namely the manifold $M=\mathbb{R}^{d} \times, \mathbb{T}$ with the metric $\mathrm{d} s^{2}=\mathrm{d} x_{1}^{2}+\cdots+\mathrm{d} x_{d}^{2}+f(x) \mathrm{d} \theta^{2}$, where $f$ is any smooth function on $\mathbb{R}^{d}$ which coincides with $\mathrm{e}^{-|x|}$ outside a compact neighbourhood of the origin.

\section{NOTATION}

Let $M$ be a complete $n$-dimensional Riemannian manifold with Riemannian metric $g$, which we assume to be smooth. We shall consider functions on $M$ and, more generally, sections of the bundle $\Lambda^{k}$ of $k$-forms on $M$. If $x$ is a point in $M$ we shall denote by $\langle\cdot, \cdot\rangle_{x}$ the canonical inner product on $\Lambda_{x}^{k}$ induced by the metric and by $|\cdot|_{x}$ the corresponding norm. We shall use standard notation and concepts from the differential and integral calculus on $M$, including the exterior derivative $\mathrm{d}$, the gradient grad and the canonical measure $\mathrm{d} V(x)=\sqrt{\operatorname{det} g(x)} \mathrm{d} x$. For $1 \leqslant p \leqslant \infty$ we denote by $L^{p}$ and $\vec{L}^{p}$ the Lebesgue spaces of functions and 1-forms, respectively, with respect to this measure. The divergence is the formal adjoint of the the exterior derivative $d$, that is, the operator div mapping $k+1$-forms to $k$-forms defined by

$$
\int_{M}\langle\operatorname{div} \omega, \eta\rangle_{x} \mathrm{~d} V(x)=-\int_{M}\langle\omega, \mathrm{d} \eta\rangle_{x} \mathrm{~d} V(x)
$$


for all smooth $k+1$-forms $\omega$ and all smooth $k$-forms $\eta$ with compact support.

The Laplace-Beltrami operator is the operator defined on smooth functions with compact support by

$$
\Delta f=-\operatorname{div} \mathrm{d} f
$$

The de Rahm Laplacian is the operator defined on smooth forms with compact support by

$$
\vec{\Delta} \omega=-(\operatorname{div} \mathrm{d}+\mathrm{d} \operatorname{div}) \omega .
$$

Since $M$ is complete, the operators $\Delta$ and $\vec{\Delta}$ are essentially selfadjoint on $L^{2}$ and on $\vec{L}^{2}$, respectively (see $[8,2])$. With an abuse of notation we denote by $\Delta$ and $\vec{\Delta}$ their unique selfadjoint extensions. We denote by $\left(P_{t}\right)_{t>0}$ and $\left(\vec{P}_{t}\right)_{t>0}$ the heat semigroup generated by $\Delta$ and $\vec{\Delta}$, respectively. The semigroup $P_{t}$ is sub-Markovian. In particular $P_{t}$ is a contraction on $L^{p}$ for every $p$ in $[1, \infty]$.

The following proposition is a special case of a result of Bakry [2, Proposition 1.7].

PROPOSITION 2.1. Let $M$ be a complete connected Riemannian manifold and denote by $T(M)$ the tangent bundle on $M$. If

$$
\operatorname{Ric}(X, X) \geqslant-K^{2}|X| \quad \forall X \in T(M),
$$

then

(i) $\left|\vec{P}_{t} \omega\right| \leqslant e^{K^{2} t} P_{t}|\omega|$ for all $\omega \in \vec{L}^{2}$ and all $t \geqslant 0$.

(ii) $\vec{P}_{t} \mathrm{~d} f=\mathrm{d} P_{t} f$ for all $f \in C_{c}^{\infty}$ and all $t \geqslant 0$.

\section{Functions of bounded VARIATION Via heat KerNel}

Denote by $\Gamma_{c}\left(\Lambda^{k}\right)$ the space of smooth $k$-forms with compact support on $M$. Given a real-valued function $u$ in $L^{1}(M)$, following [7], we define the variation of $u$ by

$$
|D u|(M)=\sup \left\{\int_{M} u \operatorname{div} \omega \mathrm{d} V: \omega \in \Gamma_{c}\left(\Lambda^{1}\right),\|\omega\|_{\infty} \leqslant 1\right\} .
$$

We say that a function $u$ in $L^{1}(M)$ has bounded variation if $|D u|(M)<+\infty$. We denote by $B V(M)$ the space of all functions in $L^{1}(M)$ with bounded variation.

LEMMA 3.1. Denote by $\Gamma_{\mathrm{bd}}\left(\Lambda^{1}\right)$ the space of all smooth bounded 1-forms with bounded divergence. Then for every $u$ in $L^{1}(M)$

$$
|D u|(M)=\sup \left\{\int_{M} u \operatorname{div} \omega \mathrm{d} V: \omega \in \Gamma_{\text {bd }}\left(\Lambda^{1}\right),\|\omega\|_{\infty} \leqslant 1\right\} .
$$

Proof: The right hand side is obviously larger than $|D u|(M)$. To prove the opposite inequality pick a sequence $\left(\phi_{n}\right)$ of functions in $C_{c}^{\infty}(M)$ such that 
(a) $0 \leqslant \phi_{n} \leqslant 1$ for all $x$ in $M$ and all $n$ in $\mathbb{N}$;

(b) for every compact set $K \subset M$ there exists $n_{K}$ such that $\phi_{n}=1$ on $K$ if $n \geqslant n_{K}$

(c) $\left\|\operatorname{grad} \phi_{n}\right\|_{\infty} \rightarrow 0$ as $n \rightarrow \infty$.

Such a sequence exists on any complete Riemannian manifold by [9, Proposition 4.1]. If $\omega \in \Gamma_{\text {bd }}\left(\Lambda^{1}\right)$ we have that $\left\|\phi_{n} \omega\right\|_{\infty} \leqslant\|\omega\|_{\infty}$ and

$$
\operatorname{div}\left(\omega \phi_{n}\right)=\phi_{n} \operatorname{div} \omega+\omega\left(\operatorname{grad} \phi_{n}\right) .
$$

Therefore, if $\omega \in \Gamma_{\mathrm{bd}}\left(\Lambda^{1}\right)$ and $\|\omega\|_{\infty} \leqslant 1$, by the dominated convergence theorem

$$
\int_{M} u \operatorname{div} \omega \mathrm{d} V=\lim _{n} \int_{M} u \operatorname{div}\left(\phi_{n} \omega\right) \mathrm{d} V \leqslant|D u|(M) .
$$

THEOREM 3.2. Let $M$ be a complete connected Riemannian manifolds with Ricci curvature bounded from below. Then for every $u$ in $L^{1}(M)$

$$
|D u|(M)=\lim _{t \rightarrow 0} \int_{M}\left|\mathrm{~d} P_{t} u\right| \mathrm{d} V
$$

Proof: First we prove that

$$
|D u|(M) \leqslant \liminf _{t \rightarrow 0}\left\|\mathrm{~d} P_{t} u\right\|_{1} .
$$

Indeed, if $\omega \in \Gamma\left(\Lambda^{1}\right)_{c}$ and $\|\omega\|_{\infty} \leqslant 1$ then, since $P_{t} u$ converges to $u$ in $L^{1}$ when $t$ tends to 0 ,

$$
\int_{M} u \operatorname{div} \omega \mathrm{d} V=\lim _{t \rightarrow 0} \int_{M} P_{t} u \operatorname{div} \omega \mathrm{d} V=-\lim _{t \rightarrow 0} \int_{M}\left\langle\mathrm{~d} P_{t} u, \omega\right\rangle \mathrm{d} V \leqslant \liminf _{t \rightarrow 0}\left\|\mathrm{~d} P_{t} u\right\|_{1} .
$$

by the dominated convergence theorem. This proves (3.2).

Next we prove that

$$
\limsup _{t \rightarrow 0}\left\|\mathrm{~d} P_{t} u\right\|_{1} \leqslant|D u|(M) .
$$

Let $\omega$ be a form in $\Gamma_{c}\left(\Lambda^{1}\right)$ such that $\|\omega\|_{\infty} \leqslant 1$. We claim that $\vec{P}_{t} \omega$ is in $\Gamma_{\text {bd }}\left(\Lambda^{1}\right)$ and $\left\|\vec{P}_{\mathrm{t}} \omega\right\|_{\infty} \leqslant \mathrm{e}^{K^{2} t}$. Indeed $\vec{P}_{t} \omega$ is smooth, because the operator $\vec{\Delta}$ is elliptic. Moreover, by Proposition $2.1\left\|\vec{P}_{t} \omega\right\|_{\infty} \leqslant \mathrm{e}^{K^{2} t}\|\omega\|_{\infty}$. Finally, since both $P_{t}$ and $\vec{P}_{t}$ are self-adjoint, part (ii) of Proposition 2.1 implies that div $\vec{P}_{t} \omega=P_{t} \operatorname{div} \omega$. Thus

$$
\left\|\operatorname{div} \vec{P}_{t} \omega\right\|_{\infty}=\left\|P_{t} \operatorname{div} \omega\right\|_{\infty} \leqslant\|\operatorname{div} \omega\|_{\infty}<\infty .
$$

because $P_{t}$ is sub-Markovian. This concludes the proof of the claim. 
Hence by Lemma 3.1 we have that

$$
\left|\int_{M} u \operatorname{div} \vec{P}_{t} \omega \mathrm{d} V\right| \leqslant e^{K^{2} t}|D u|(M)
$$

Therefore

$$
\begin{aligned}
\left|\int_{M}\left\langle\mathrm{~d} P_{t} u, \omega\right\rangle \mathrm{d} V\right| & =\left|\int_{M} P_{t} u \operatorname{div} \omega \mathrm{d} V\right| \\
& =\left|\int_{M} u P_{t} \operatorname{div} \omega \mathrm{d} V\right| \\
& =\left|\int_{M} u \operatorname{div} \vec{P}_{t} \omega \mathrm{d} V\right| \\
& \leqslant e^{K^{2} t}|D u|(M) .
\end{aligned}
$$

Taking the supremum with respect to all $\omega$ in $\Gamma_{c}\left(\Lambda^{1}\right)$ with $\|\omega\|_{\infty} \leqslant 1$ in the left side, we obtain

$$
\left\|\mathrm{d} P_{t} u\right\|_{1} \leqslant e^{K^{2} t}|D u|(M) .
$$

Passing to the limit as $t$ tends to 0 we get (3.3). Theorem 3.2 now follows by combining (3.2) and (3.3).

\section{REFERENCES}

[1] L. Ambrosio, N. Fusco and D. Pallara, Functions of bounded variation and free discontinuous problems, Oxford Mathematical Monographs (The Clarendon Press Oxford University Press, New York, 2000).

[2] D. Bakry, 'Études des transformations de Riesz dans les variétés riemanniennes à courbure de Ricci minorée', Lecture Notes in Maths 1247 (Springer-Verlag, Verlin).

[3] E. De Giorgi, 'Su una teoria generale della misura $(r-1)$-dimensionale in uno spazio ad r dimensioni', Ann. Mat. Pura Appl. 36 (1954), 191-213.

[4] E. De Giorgi, Selected Papers, (L. Ambrosio, G. Dal Maso, M. Forti, M. Miranda, S.Spagnolo, Editors) (Springer Verlag, Berlin, Heidelberg, 2006).

[5] G. Fichera, Lezioni sulle trasformazioni lineari, Istituto Matematico dell'Università di Trieste, Vol. I, 1954.

[6] M. Miranda, 'Distribuzioni aventi derivate misure. Insiemi di perimetro localmente finito', Ann. Scuola Norm. Sup. Pisa Cl. Sci. 18 (1964), 27-56.

[7] M. Miranda Jr., D. Pallara, F. Paronetto and M. Preunkert, 'Heat semigroup and functions of bounded variation on Riemannian manifolds', (preprint (2005) cvgmt.sns.it/papers/mirpalpar05a/).

[8] R.S. Strichartz, 'Analysis of the Laplacian on the complete Riemannian manifold', $J$. Funct. Anal. 52 (1983), 48-79.

[9] M. Shubin, 'Essential self-adjointness for semi-bounded magnetic Schrödinger operators on non-compact manifolds', J. Funct. Anal. 186 (2001), 92-116. 
Dipartimento di Matematica

Università di Genova

via Dodecaneso 35

16146 Genova

Italy 